\title{
Carrier and Sampling Frequency Offset Estimation and Tracking in OFDM Systems
}

\author{
Adrian Langowski
}

Original scientific paper

\begin{abstract}
In This paper presents the proposition of the tracking algorithm of the carrier frequency and sampling frequency offsets. The algorithm uses a phase difference between channel transfer function estimates computed for the same subcarrier of two successive OFDM symbols. Proposed solution was compared, by means of computer simulation, with similar non-data aided algorithm introduced in [1]. Both schemes were tested by transmitting OFDM symbols over the Rayleigh fading channel.
\end{abstract}

\section{INTRODUCTION}

Due to many advantages Orthogonal Frequency Division Multiplexing (OFDM) was adopted for the European standards of terrestrial stationary and handheld video broadcasting systems (DVB-T, DVB-H) and wireless network standards 802.11 and 802.16. It was also chosen as one of the transmission techniques for WINNER Radio Interface Concept [2] which has been recently proposed for $4 \mathrm{G}$ systems. However, the OFDM transmission is sensitive to receiver synchronization imperfections. The symbol time synchronization error may cause Inter-Block Interference (IBI) and the frequency synchronization error is the mainspring of Inter-Carrier Interference (ICI). In contrast to constant symbol timing offset constant carrier frequency offset (CFO) causes variable phase errors. The uncompensated $\mathrm{CFO}$ equal to $1 \%$ of the subcarrier spacing may change completely the signal polarity after several dozen of received OFDM symbols [3].

Regardless the form of the OFDM transmission (whether it is continuous or has a bursty nature) the sampling frequency offset (SFO) cannot be neglected. The inaccuracy of the sampling clock causes slow drift of the FFT window causing ICI and subcarrier phase rotation. Both signal distortions, but not their source, may be removed by a channel equaliser. However, the time shift of FFT window caused by the sampling period offset builds up and finally the FFT window shifts beyond the orthogonality window of the OFDM symbol giving rise to IBI. Therefore the sampling clock synchronization, also called re-sampling algorithm, should also be implemented in the OFDM receiver.

In order to remove the degrading influence of the carrier frequency and sampling frequency inaccuracy a number of

The material in this paper was presented in part at the $16^{\text {th }}$ International Conference on Software, Telecommunications and Computer Networks (SoftCOM 2008), Split - Dubrovnik, Croatia, Sept. 2008.

Adrian Langowski is with the Poznan University of Technology, Poznan, Poland. algorithms have been already proposed. The best known method of CFO tracking, proposed by Beek in [4], performs a correlation between the cyclic prefix and the corresponding end of the OFDM symbol. No pilot tones are required, what decreases redundancy, however, the estimates quality depends on the accuracy of the symbol timing synchronisation. In contrast to Beek's method most tracking algorithms perform in the frequency domain. Within this group of algorithms subcarrier phase rotation caused by CFO and SPO is exploited. Some of them, like the algorithms introduced in [5, $6]$, estimate its change between the subcarriers of the OFDM symbol or between the same subcarriers of succeeding OFDM symbols (see method described in [1]). The noncoherent solution, i.e., without carrier phase estimates, was proposed in [7], however, the algorithm can estimate SFO only. The drawback of the algorithm [7] is its sensitivity to symbol timing synchronisation errors. Similarly to the schemes shown in [5] and [6] it requires pilot tones transmitted in every OFDM symbol, as it is done in the DVB-T system. Thus, such algorithms are not suitable for systems with pilot tones separated in time by data symbols, as it can be found in the WINNER system. The algorithm described in paper [1] is driven by data hard decisions made by the receiver, however, fails to correctly estimate CFO and SFO offsets in low SNRs.

In this paper the author proposes a low complex, immune to symbol timing errors, carrier frequency and sampling frequency offsets estimator. The algorithm is designed for the OFDM systems with small pilot overhead and it uses channel transfer function estimates already computed by the receiver's channel estimation block.

The paper is organised as follows. In Section II the system model is introduced. In Section III timing synchronisation errors are briefly characterised. Sections IV and V contain the description of the decision-directed algorithm and newly proposed algorithm in which channel transfer function estimates are used. Computer simulation results are presented and discussed in Section VI and finally the paper is concluded in Section VII.

\section{SYSTEM MODEL}

The system of interest uses OFDM symbols with $K U<N$ subcarriers for data transmission. The remaining $N-K U$ subcarriers serve as a guard band. The time domain samples are computed with the well known IFFT formula

$$
x_{k}(n)=\frac{1}{\sqrt{N}} \sum_{i=0}^{K_{U}-1} X_{k}(i) e^{j \omega_{N} i n}
$$




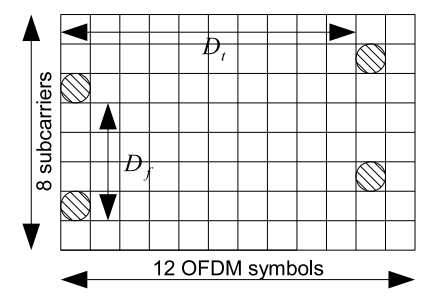

Figure 1 - Pilot tones pattern within the chunk

where $k$ is the index of the OFDM symbol, $X_{k}(i)$ is the $i$-th modulated symbol in frequency domain, $\omega_{N}=2 \pi / N$, and $N$ is the total number of subcarriers. Let us assume the OFDM signal model developed within the WINNER project [2]. The user data are transmitted in packets called chunks. Every chunk consists of 8 subcarriers and lasts for 12 OFDM symbols. Within each chunk there are 4 pilot tones spaced by $D_{t}=10$ OFDM symbols and by $D_{f}=4$ subcarriers [8]. Their pattern is shown in Figure 1. Generated OFDM symbols are grouped into packets and transmitted over multipath channel with Rayleigh fading channel for which the impulse response is

$$
h(\tau, t)=\sum_{l=0}^{L-1} h_{l}(t) \delta\left(\tau-\tau_{l}\right)
$$

where $h_{l}(t)$ is a complex channel coefficient of the $l$-th path, $\tau_{l}$ is a delay of the $l$-th path and $L$ is the number of channel paths.

\section{SYNCHRONISATION ERRORS}

Assuming the timing synchronisation was successful enough to find the OFDM symbol start within IBI-free region, two kinds of frequency offsets remain after the acquisition mode, i.e. sampling frequency offset (SFO) and residual carrier frequency offset (CFO). Denote $\varepsilon=\left(T_{s}^{\prime}-T_{s}\right) / T_{s}$ as the normalised SFO and $\delta f_{N}=\delta f / \Delta f$ as the normalised frequency offset, where $T_{s}^{\prime}, T_{s}, \delta f$ and $\Delta f$ are erroneous and error-free sampling periods, carrier frequency offset and subcarrier distance, respectively. The data symbol received on the $m$-th subcarrier of the $k$-th OFDM symbol is described by $[1,9,10]$

$$
\begin{aligned}
Y_{k}(m)= & \alpha(\theta(m)) X_{k}(m) H_{k}(m) e^{j \pi \theta(m)(N-1) / N} e^{j 2 \pi \theta(m)\left(N_{G}+k M\right) / N} \\
& +I C I_{k}(m)+N_{k}(m)
\end{aligned}
$$

where $\theta(m)=\delta f_{N}(1+\varepsilon)+m \varepsilon \approx \delta f_{N}+m \varepsilon, M=N+N_{G}$, $\alpha(\theta(m))$ is an attenuation caused by both offsets and $N_{k}(m)$ is the Gaussian noise sample.

The sampling period offset affects the OFDM signal twofolds. Firstly, it rotates data symbols, secondly, since accumulated sampling period offset is not constant during the OFDM symbol but is rises from sample to sample, it disturbs the orthogonality of the subcarriers giving rise to intercarrier interference. However, for small offsets the second phenomenon and the attenuation are negligible and they will not be considered in this work.

\section{DECISION-DIRECTED ALGORITHM}

Decision-directed estimation of the sampling period offset and carrier frequency offset was proposed in [1] and is presented as a reference to the proposed method. First the phasedifference dependent signal $\lambda_{k}^{D D}(m)$ for each subcarrier is computed

$$
\lambda_{k}^{D D}(m)=\frac{Y_{k}(m) Y_{k-1}^{\star}(m)}{\tilde{D}_{k}(m) \tilde{D}_{k-1}^{\star}(m)}
$$

where $\tilde{D}_{k}(m)$ is the hard data decision, and $(.)^{\star}$ denotes the complex conjugate. The arguments of the above signals are then used for CFO and SFO estimation

$$
\begin{gathered}
\delta \tilde{f}_{N_{k}}=\frac{\rho}{2 \pi} \frac{\varphi_{k, 1}+\varphi_{k, 2}}{2} \\
\tilde{\varepsilon}_{k}=\frac{\rho}{2 \pi} \frac{\varphi_{k, 2}-\varphi_{k, 1}}{\frac{K_{U}}{2}+1}
\end{gathered}
$$

where

$$
\varphi_{k, 1}=\arg \left(\sum_{i \in \mathbb{C}_{1}} \lambda_{k}^{D D}(i)\right) \varphi_{k, 2}=\arg \left(\sum_{i \in \mathbb{C}_{2}} \lambda_{k}^{D D}(i)\right)
$$

where $\mathbb{C}_{1}=\left\langle-K_{U} / 2,-1\right\rangle$ and $\mathbb{C}_{2}=\left\langle 1, K_{U} / 2\right\rangle$ are the sets of indices of the first and the second half of the OFDM symbol subcarriers, respectively and $\rho=N / M$. The one-shot estimates are filtered using the first-order tracking loop filters

$$
\begin{aligned}
\delta \hat{f}_{N_{k}} & =\delta \hat{f}_{N_{k-1}}+\gamma_{f} \delta \tilde{f}_{N_{k}} \\
\hat{\varepsilon}_{k} & =\hat{\varepsilon}_{k-1}+\gamma_{\varepsilon} \tilde{\varepsilon}_{k}
\end{aligned}
$$

where $\gamma_{f}$ and $\gamma_{e}$ are CFO and SFO loop filters coefficients, respectively. The sampling period offset estimate controls the interpolator/decimator block that corrects the offset. The carrier frequency offset is used for correcting the phase of time samples of the received OFDM signal. The drawback of this algorithm is that CFO estimate does not take into consideration the influence of SFO that can be significant during the initialisation of the algorithm.

\section{PROPOSED ALGORITHM}

The phase rotation of the subcarrier is easily detectable by the channel estimator and is estimated jointly with the channel transfer function. Thus, the generalised CTF takes the form

$$
H_{k}^{\prime}(m)=H_{k}(m) e^{j \pi \theta(m)(N-1) / N} e^{j 2 \pi \theta(m)\left(N_{G}+k M\right) / N}
$$

The author proposes to apply the knowledge obtained by the channel estimator for sampling period offset correction. The phase-difference-dependent $\lambda_{k}(m)$ is defined as follows

$$
\lambda_{k}(m)=\widetilde{H}_{k}^{\prime}(m) \widetilde{H}_{k-1}^{\prime \star}(m)
$$

where $\widetilde{H}_{k}(m)$ is the CTF estimate of the $m$-th channel. In the proposed scheme no interpolator/decimator block is used but carriers phases are corrected instead. This implies that the intercarrier interference remains unchanged, however, the receiver is simpler and thus cheaper. Another consequence of 
this solution is that the FFT window drift during one OFDM symbol is estimated instead of exact sampling period offset. After substituting (9) into (10) and modifying the intermediate result the phase-difference-dependent $\lambda_{k}(m)$, assuming $H_{k}(m) \approx H_{k-1}(m)$ is defined as

$$
\lambda_{k}(m)=\left|\widetilde{H}_{k}^{\prime}(m)\right|^{2} e^{j 2 \pi\left(\delta f_{N}+\varepsilon m\right) / \rho}
$$

The phase rotation of the subcarrier $m$ depends on the CFO, SFO and the subcarrier index. In order to estimate the sampling frequency offset the influence of subcarrier index $m$ is removed by computing the product of complex conjugate signal received on subcarrier $m$ and signal received $K_{U} / 1+1$ subcarriers apart, i.e. in the second half of the OFDM symbol bandwidth. One-shot sampling frequency offset estimate is given by

$$
\tilde{\varepsilon}_{M, k}=M \tilde{\varepsilon}_{k}=\frac{N}{2 \pi} \frac{\varphi_{\varepsilon, k}}{\frac{K_{U}}{2}+1}
$$

where

$$
\begin{aligned}
\varphi_{\varepsilon, k} & =\arg \left(\sum_{i \in \mathbb{C}_{1}} \lambda_{k}\left(i+\frac{K_{U}}{2}+1\right) \lambda_{k}^{\star}(i)\right) \\
& \approx \frac{2 \pi}{\rho} \varepsilon\left(\frac{K_{U}}{2}+1\right)
\end{aligned}
$$

$\mathbb{C}_{1}$ is the set of indices of the pilot subcarriers in the first half of the OFDM symbol. The approximation in (13) becomes exact if the channel transfer function estimate samples $\tilde{H}_{k}(m),(m=1, \ldots, N)$ are ideal and there is no additive noise. In order to estimate the carrier frequency offset, first the phase $\varphi_{f, k}$ is computed

$$
\begin{aligned}
\varphi_{f, k} & =\arg \left(\sum_{i \in \mathbb{C}_{1}} \lambda_{k}\left(i+\frac{K_{U}}{2}+1\right) \lambda_{k}(i)\right) \\
& \approx \frac{2 \pi}{\rho} 2 \delta f_{N}+\frac{2 \pi}{\rho} \varepsilon\left(\frac{K_{U}}{2}+1\right)+N_{k}
\end{aligned}
$$

where

$$
N_{k}=\arg \sum_{i \in \mathbb{C}_{1}} e^{j \frac{2 \pi}{\rho} 2 \varepsilon i}\left|\tilde{H}_{k}^{\prime}\left(i+\frac{K_{U}}{2}+1\right)\right|^{2}\left|\tilde{H}_{k}^{\prime}(i)\right|^{2}
$$

can be interpreted as a phase noise caused by the sampling frequency offset. It can be seen that the second component in (14) is equal to the phase (13) and in this case is undesired. Thus, the one-shot CFO estimate is given by

$$
\delta \tilde{f}_{N, k}=\frac{\rho}{2 \pi} \frac{\varphi_{f, k}-\varphi_{\varepsilon, k}}{2}
$$

Both sampling frequency offset estimate $\tilde{\varepsilon}_{M, k}$ and carrier frequency offset estimate $\delta \tilde{f}_{N, k}$ are fed to two second-order digital phase-locked loop (DPLL) filters whose block diagram is presented in Figure 2. Coefficients $\mu_{1}$ and $\mu_{2}$ are integral and proportional coefficients, respectively. The transfer function of the DPLL is [11]

$$
\begin{aligned}
H(z) & =\frac{\mu_{2}(z-1)+\mu_{1}}{(z-1)^{2}+\mu_{2}(z-1)+\mu_{1}} \\
& =\frac{2 \zeta \omega_{n}(z-1)+\omega_{n}^{2}}{(z-1)^{2}+2 \zeta \omega_{n}(z-1)+\omega_{n}^{2}}
\end{aligned}
$$

where $\mu_{2}=2 \zeta \omega_{n} T_{s}, \mu_{1}=\mu_{2}^{2} / 4 \zeta^{2}, \omega_{n}=2 \pi f_{n}, T_{s}$ is the sampling period, $\zeta$ is the damping factor and $f_{n}$ is the natural frequency of the loop. In order to guarantee the stability of the loop the damping factor $\zeta$ and the natural frequency $f_{n}$ must satisfy the following relationship [12]

$$
\left\{\begin{array} { l } 
{ \zeta > 1 } \\
{ 0 < \omega _ { n } < 2 } \\
{ \zeta \omega _ { n } < ( \omega _ { n } ^ { 2 } / 4 ) + 1 }
\end{array} \text { or } \left\{\begin{array}{l}
\zeta \leq 1 \\
0<\omega_{n}<2 \zeta
\end{array}\right.\right.
$$

The integer $\hat{\varepsilon}_{\text {int }}$ and fractional $\hat{\varepsilon}_{\text {fra }}$ parts of the accumulated sampling period error are extracted from the sampling frequency offset loop output $\hat{\varepsilon}_{M, k}$. The integer part is used for FFT window shift while the fractional one is used for correcting the subcarriers phase.

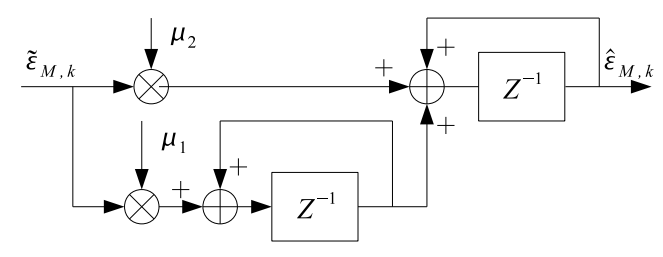

Figure 2 - Second-order digital phase-locked loop filter diagram

In the proposed CFO and SFO estimation algorithms an estimation of the channel transfer function is needed. In our design the Least Squares (LS) channel estimator was applied to obtain the initial channel estimate [13]

$$
\tilde{H}_{1}(m)=\frac{\tilde{D}_{i}(m) Y_{1}^{\star}(m)}{\left|\tilde{D}_{i}(m)\right|^{2}}
$$

The symbol $\tilde{D}_{i}(m)$ is the hard decision made by the demodulator, however, when the first OFDM symbol of the superframe is received the symbol represents the pilot symbol known to the receiver. After receiving the first OFDM symbol the estimator switches to the tracking mode. The channel estimates are refined and tracked according to the gradient algorithm minimising the mean square error [14]

$$
\begin{aligned}
\tilde{H}_{k+1}(m)= & \tilde{H}_{k}(m)+ \\
& +\delta\left(Y_{k}(m)-\tilde{H}_{k}(m) \tilde{D}_{k}(m)\right) \tilde{D}_{k}^{\star}(m)
\end{aligned}
$$

where $\delta$ is the coefficient dependent on the transmitted symbols power and is constant during the transmission. The channel coefficients are updated every received OFDM symbol. The author would like to stress that the channel estimator is not an integral part of proposed scheme and other channel estimation algorithms can be applied as well. 


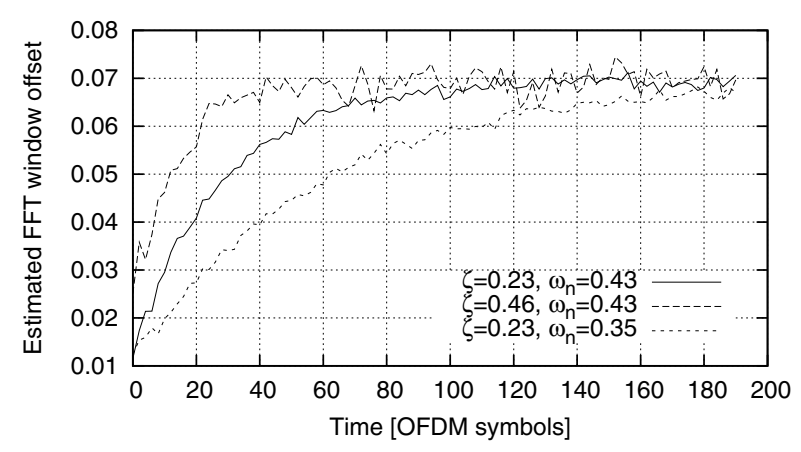

Figure 3 - The influence of loop parameters on its convergence speed

\section{SIMULATION RESULTS}

The proposed sampling period offset estimation algorithm and the decision-directed solution were tested for the following WINNER system parameters, i.e., the size of IFFT $N=2048$, the number of used subcarriers $K_{U}=1152$, the cyclic prefix length $N_{G}=256, \Delta f=39062.5 \mathrm{~Hz}$, the carrier frequency $f_{c}=3.95 \mathrm{GHz}$ and the number of OFDM symbols in the packet equal to 192 .

The channel was simulated using the 20-path, Rayleigh fading NLOS channel model with the root-mean square delay spread $\tau_{R M S}$ of $310 \mathrm{~ns}$. This model was developed within the WINNER project for Typical Urban (TU) environment [15]. The terminal moved with the maximal velocity equal to $70 \mathrm{~km} / \mathrm{h}$. Both user data and pilots were mapped onto QPSK constellation. The simulation results were obtained using 10000 channel realisations for each $S N R$ value and the algorithm performance was tested for sampling period offset $\delta T_{s}$ equal to 5 and $30 \mathrm{ppm}$.

The important step of developing the tracking algorithms is the choice of the loop parameters $\zeta$ and $\omega_{n}$. Increasing the damping factor or natural frequency decreases the convergence speed, as it is illustrated in Figure 3. However, attention should be paid while choosing the parameters, since mean square error (MSE) of the filtered output increases with damping factor and decreases with natural frequency, as it is presented in Figure 4. The first curve was generated for fixed $\omega_{n}$ and variable $\zeta$ and the second one for fixed $\zeta$ and variable $\omega_{n}$. After preliminary tests the following loops parameters were chosen. For the DD and the proposed algorithms $\zeta=0.229$ and $\omega_{n}=0.436$ for the sampling frequency offset tracking loop. For the carrier frequency offset tracking loop $\zeta=0.4, \omega_{n}=0.5$ for the DD algorithm, and for the proposed algorithm $\zeta=0.3$ and $\omega_{n}=0.5$.

The carrier frequency and sampling frequency offsets estimation and tracking algorithms were tested for the frequency offset $\delta f=0.05$ and the sampling frequency offset $\delta T_{s}=5 \mathrm{ppm}$ and $30 \mathrm{ppm}$. The results of SFO estimation by the proposed method are illustrated in Figure 5. The MSE of the estimated sampling period offset is the same, except for small signal power, for both small and large offsets. The larger, with respect to the offset value, MSE for small offsets

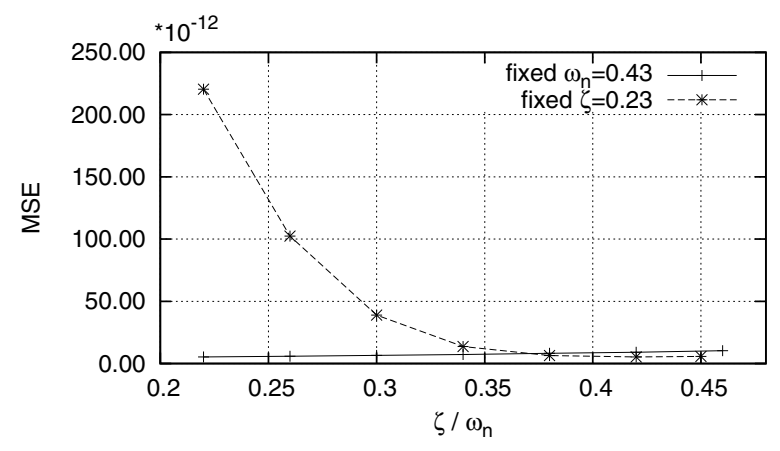

Figure 4 - The influence of loop parameters on MSE

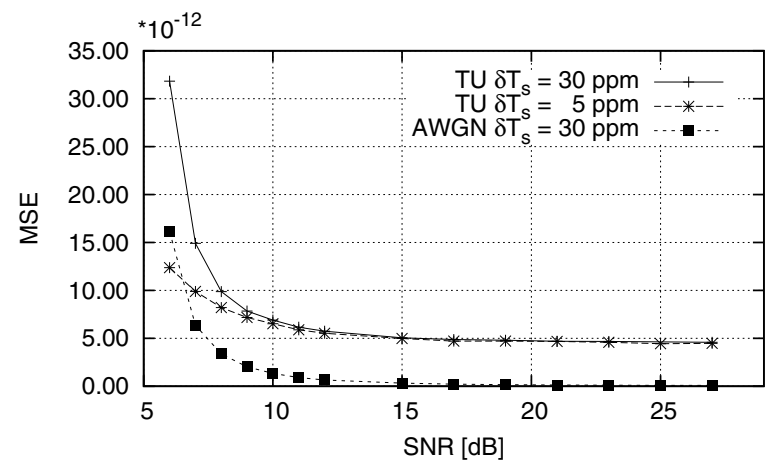

Figure 5 - The mean square error of the estimated SFO by the proposed solution for different $\delta T_{s}$ and $\delta f=0.05$

is related to the limited accuracy of the implemented channel estimator. Its influence on the algorithm performance is visible when compared with the results achieved for an AWGN channel only. The channel estimation errors increase MSE of the sampling period offset estimate 40 times. Exactly the same behaviour of the proposed algorithm can be observed during the estimation of the carrier frequency offset (Figure 6). Both algorithms, i.e. the proposed and decisiondirected ones were compared in the TU channel for sampling period offset $\delta T_{s}=30 \mathrm{ppm}$ and $\delta f=0.05$. However, similarly to the proposed solution the DD algorithm was modified to estimate the accumulated FFT window shift caused bu SFO instead of estimating SFO only. Moreover, carrier and sampling frequency offsets were filtered using secondorder DPLL. Both solutions used the same sets of subcarrier indices $\mathbb{C}_{1}$ and $\mathbb{C}_{2}$. The results plotted in Figures 8 and 7 indicate that the proposed algorithm deals better with severe channel conditions than the decision-directed one. Worse performance of the DD algorithm is related to the channel estimate phase error amplification by the hard-decision data demodulator and its propagation to the phase-differencedependent signal (4). Since in the proposed solution no harddecisions are used the phase errors of the erroneous channel estimates are not amplified and their influence on the overall algorithm performance is smaller than in the DD algorithm. 


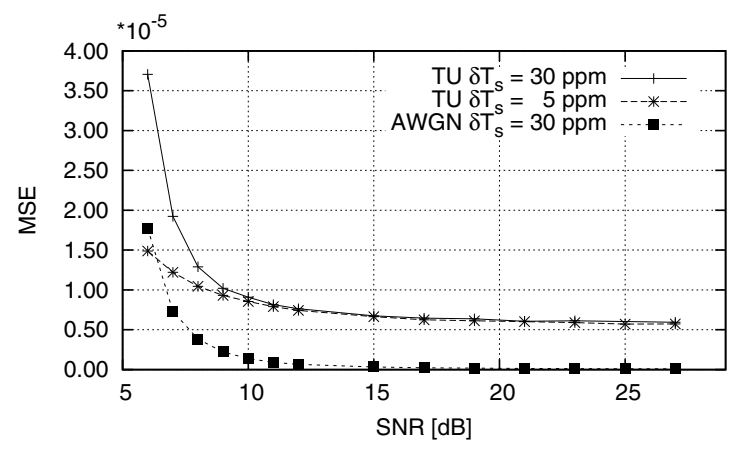

Figure 6 - The mean square error of the estimated $C F O$ by the proposed algorithm for different $\delta T_{s}$ and $\delta f=0.05$

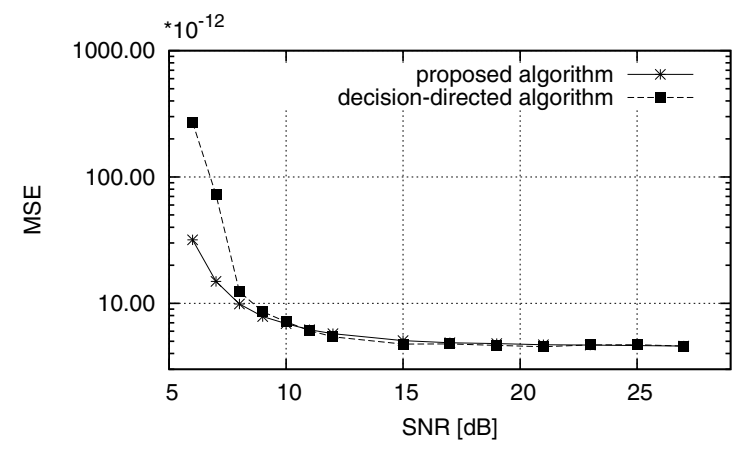

Figure 7 - The mean square error of the estimated SFO for $\delta T_{s}=30 \mathrm{ppm}$ and $\delta f=0.05$

\section{CONCLUSIONS}

In this paper the new sampling period offset estimation algorithm has been introduced. The algorithm is based on the channel estimates already computed by the channel estimator and is designed for transmission systems with low pilot overhead. The complexity of the proposed solution is slightly lower than of the decision-directed one. The algorithm was tested by means of computer simulations in TU environment, giving smaller MSE of the CFO and SFO estimates than the algorithm of reference.

\section{REFERENCES}

[1] K. Shi, E. Serpedin, and P. Ciblat, "Decision-Directed Fine Synchronisation in OFDM Systems," IEEE Trans. Commun., vol. 53, no. 3, pp. 408-412, Mar. 2005.

[2] D2.10 Final Report on Identified RI Key Technologies, System Concept, and Their Assessment, IST-2003507581 WINNER, December 2005.

[3] H. Tang, K. Y. Lau, and R. W. Brodersen, "Synchronization Schemes for Packet OFDM System," in Proc. IEEE International Conference on Communications (ICC'03), vol. 5, May 2003, pp. 3346-3350.

[4] J. J. van de Beek, M. Sandell, and P. Börjesson, "ML Estimation of Time and Frequency Offset in OFDM Systems," IEEE Trans. Signal Process., vol. 45, no. 7, pp. 1800-1805, Jul. 1997.

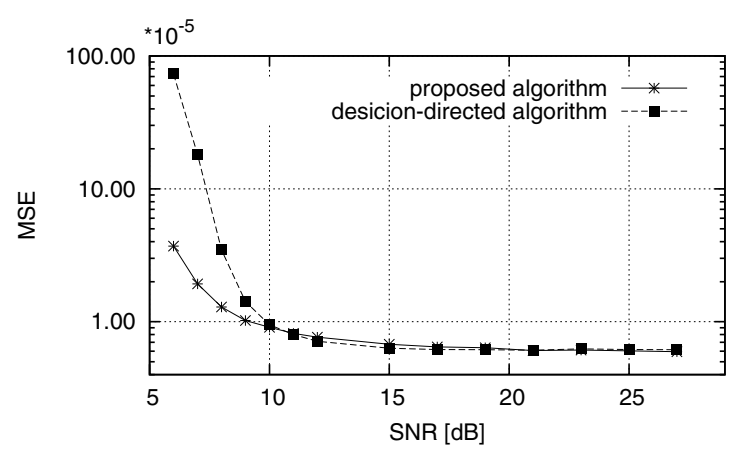

Figure 8 - The mean square error of the estimated $C F O$ for $\delta f=0.05$ and $\delta T_{s}=30 \mathrm{ppm}$

[5] D. K. Kim, S. H. Do, H. B. Cho, H. J. Choi, and K. B. Kim, "A New Joint Algorithm of Symbol Timing Recovery and Sampling Clock Adjustment for OFDM Systems," IEEE Trans. Consum. Electron., vol. 44, no. 3, pp. 1142-1149, Aug. 1998.

[6] S. A. Fechtel, "OFDM Carrier and Sampling Frequency Synchronisation and its Performance on Stationary and Mobile Channels," IEEE Trans. Consum. Electron., vol. 46, no. 3, pp. 438-441, Aug. 2000.

[7] B. Yang, K. B. Letaief, R. S. Cheng, and Z. Cao, "Timing Recovery for OFDM Transmission," IEEE J. Sel. Areas Commun., vol. 18, no. 11, pp. 2278-2291, Nov. 2000.

[8] Link level procedures for the WINNER System, IST-4027756 WINNER II, November 2007.

[9] P. Moose, "A Technique for Orthogonal Frequency Division Multiplexing Frequency Offset Correction," IEEE Trans. Commun., vol. 42, pp. 2908-2914, Oct. 1994.

[10] M. Luise and R. Reggiannini, "Carrier Frequency Acquisition and Tracking for OFDM Systems," IEEE Trans. Commun., vol. 44, pp. 1590-1598, Nov. 1996.

[11] F. M. Gardner, Phaselock Techniques. John Wiley and Sons, 2005.

[12] Z.-W. Zheng, Z.-X. Yang, C.-Y. Pan, and Y.-S. Zhu, "Novel Synchronisation for TDS-OFDM-Based Digital Television Terrestial Broadcast Systems," IEEE Trans. Broadcast., vol. 50, no. 2, pp. 148-153, Jun. 2004.

[13] J. Proakis, Digital Communications, 4th ed. McGrawHill, 2001.

[14] A. Langowski, A. Piątyszek, Z. Długaszewski, and K. Wesołowski, "VHDL Realisation of the Channel Estimator and the Equaliser in the OFDM Receiver,' in Proc. X National Symposium of Radio Science, URSI 2002, March 2002, pp. 129-134.

[15] D5.4 Final Report on Link Level and System Level Channel Models, IST-2003-507581 WINNER, September 2005 . 